\title{
Adaptive Decentralized Control Scheme for a Stochastic Interconnected System
}

\author{
Xiaoli Jiang $\mathbb{D}^{1},{ }^{1}$ Siqi Liu, ${ }^{1}$ Mingyue Liu, ${ }^{1}$ Li Yang, ${ }^{1}$ and Lina Liu $\mathbb{D}^{2}$ \\ ${ }^{1}$ College of Mathematics and Physics, Bohai University, Jinzhou 121013, Liaoning, China \\ ${ }^{2}$ School of Electronic and Information Engineering, Soochow University, Suzhou, Jiangsu 215006, China
}

Correspondence should be addressed to Xiaoli Jiang; jxls309@163.com and Lina Liu; 1ln@suda.edu.cn

Received 17 April 2020; Revised 28 June 2020; Accepted 30 July 2020; Published 29 August 2020

Academic Editor: Quanxin Zhu

Copyright (c) 2020 Xiaoli Jiang et al. This is an open access article distributed under the Creative Commons Attribution License, which permits unrestricted use, distribution, and reproduction in any medium, provided the original work is properly cited.

This work investigates a decentralized state feedback scheme of neural network control for an interconnected system. The completely unknown associated terms are estimated directly by the neural structure. A modified approach is proposed to deal with the state feedback format. By combining the Lyapunov function and backstepping technology together, an adaptive decentralized controller is established, and we can construct the boundedness of all signals in the closed-loop structure through the controller, which can drive the formation of a given reference signal. In the end, the effectiveness of the presented strategy is referred to a simulation example.

\section{Introduction}

Interconnected systems are a race of large-scale systems, which contain some related subsystems. Interactions among subsystems often exist in interconnected systems, such as aerospace systems and electric power and computer systems. Decentralized control method is effective to solve stability of the interconnected structure. Its main principle is to use local information to form local controllers and achieve to control the entire system. The advantage is that it is simpler and more effective than the centralized control. Some important achievements have been made in this field, especially the stabilization and tracking control issues, see [1-7]. Planting parameters and interactions among subsystems are often unknown; the decentralized control technique via Nussbaum gain function can solve the difficulty, which only depends on local measurements [8]. In [9-11], the authors discussed some decentralized ideas to deal with the dimensional uncertainty of interconnected systems.

Recently, research on decentralized control using backstepping approach has also received considerable attention, such as improving transient performance [12]. This control strategy only uses local signals to design local controllers for each subsystem, which not only simplifies the controller structure but also improves the stability and performance analysis of the whole closed-loop system when there is uncertain interaction between subsystems. In the early stage of research, decentralized adaptive control was mainly based on the traditional deterministic equivalence principle, which usually requires some conservative assumptions on the structure and interaction of subsystems. By means of the linear state observer, several different controllers were developed by Ji et al. and Liu et al. [13, 14]. Some feedback control approaches have been presented for different systems in $[13,15,16]$. The authors in [17] solved the exponential stability criteria for interval-delayed neural networks. A filter was constructed to eliminate interference signals in [18]. The event-triggered feedback control was studied for an exogenous disturbance system [19]. In fact, stochastic disturbances often exist in the practical systems; it should be noted that the aforementioned adaptive algorithms were only limited to the uncertain ones, and they cannot be directly used in those interconnected ones with stochastic forms; this kind of problem has not been studied in depth. In addition, the control format for stochastic systems involves Itô formula which contains gradient terms and also includes higher-order Hessian terms. Therefore, how to design a stable scheme for interconnected stochastic systems is our main purpose. 
It is common knowledge that fuzzy logic or neural network structure is valid to settle the indeterminacy. Its basic conception is to select controllers by backstepping technique and then choose a system to approximate the indeterminate functions. This function estimation technique has been developed for the completely unknown nonlinearities, which has been applied to single input or output models in [20-27], and multiple input and output models were established in [28-31]. As a specialized approximator, a fuzzy logic structure was employed to estimate the unknown functions, and an output controller was established in [28]. In [29, 30], the controllers of multiple input and output models were established. Combining with the radial basis function, an adaptive feedback algorithm was proposed in [31]. The aforementioned conclusions have been extended to output feedback cases in $[22,23]$. In [32], adaptive dynamics and higher and lower powers were introduced to construct the controller, and the state feedback stabilization was obtained by using the Lyapunov function and the backstepping method. Neural networks solved the unmeasured states, and decentralized controllers were discussed in $[33,34]$. Therefore, a natural idea is that we can control interconnected systems with various uncertainties by the above methods. Although many results have been listed on various models with nonlinearities, the interaction among subsystems is unknown and satisfies the high-order nonlinear boundary, numerous actual problems cannot be converted into the ideal state, and the approximation-based inversion technology is hindered. When the system is considered as an interconnection type and wants to achieve a steady state, the existing control methods cannot deal with this type of problem. In fact, few results were reported on the stabilization for stochastic interconnect systems. In most of research studies, adaptive controllers were constructed in traditional backstepping design. Whether this method can be directly applied to obtain controllers for stochastic and interconnected characteristics, and what improvements are needed are the issues to be discussed in this work.

Inspired by the above works, this paper investigates an adaptive neural decentralized scheme for an uncertain interconnected system with unknown terms. It is supposed that only the output state can be measured, and there exist uncertain nonlinear functions, unknown associated terms, and stochastic disturbance. We design a neural controller via a modified backstepping approach. The proposed scheme is independent of the prior knowledge of the basis functions. Compared with the existing design methods, the innovation points of this paper are stated as follows:

(1) When the completely unknown associated terms exist and the state variable information is unknown, a modified adaptive neural scheme is proposed to deal with the distributed large-scale systems. It is different from the above works that the whole system needs only one virtual control signal. It is not necessary to repeatedly distinguish the virtual signals in each subsystem. The reliability can be improved when the random phenomenon occurs.
(2) Only one adaptive parameter is needed in the design process, so the proposed control method has fewer parameters and less calculation.

The rest of this work is composed of the following: we introduced some assumptions and preliminaries and described problem statements in Section 2, as well as adaptive neural controller design and stability analysis are listed Section 3. Simulation results can been found in Section 4, with conclusion in Section 5.

\section{Problem Statements and Preliminaries}

2.1. System Description. Now, let us first give our system and some related assumptions. Consider an interconnected system that is composed of $N$ subsystems; the $i^{\text {th }}$ subsystem is given as

$$
\left\{\begin{array}{l}
d x_{i, 1}=\left(x_{i, 2}+h_{i, 1}\left(\bar{x}_{i, 1}\right)+\Phi_{i, 1}(\bar{y})\right) \mathrm{d} t+\psi_{i, 1}^{T}\left(y_{i}\right) \mathrm{d} \omega_{i}, \\
d x_{i, 2}=\left(x_{i, 3}+h_{i, 2}\left(\bar{x}_{i, 2}\right)+\Phi_{i, 2}(\bar{y})\right) \mathrm{d} t+\psi_{i, 2}^{T}\left(y_{i}\right) \mathrm{d} \omega_{i}, \\
\vdots \\
d x_{i, n_{i}}=\left(u_{i}+h_{i, n_{i}}\left(\bar{x}_{i, n_{i}}\right)+\Phi_{i, n_{i}}(\bar{y})\right) \mathrm{d} t+\psi_{i, n_{i}}^{T}\left(y_{i}\right) \mathrm{d} \omega_{i}, \\
y_{i}=x_{i, 1},
\end{array}\right.
$$

where $\bar{x}_{i, j}=\left[x_{i, 1}, \ldots, x_{i, j}\right]^{T}, \quad \bar{y}=\left[y_{1}, y_{2}, \ldots, y_{N}\right]^{T}, x_{i}=\left[x_{i, 1}\right.$, $\left.x_{i, 2}, \ldots, x_{i, n_{i}}\right]^{T} \in R^{n_{i}}$, and $u_{i} \in R$ and $y_{i} \in R$ are the input and output of the system, respectively. $\psi_{i, j}\left(y_{i}\right)$ is the smooth function with $\psi_{i, j}(0)=0$, and $\omega_{i}$ denotes an $r$-dimensional standard Brownian motion defined on the complete probability space $\left(\Delta, F,\left\{F_{t}\right\}_{t \geq 0}, P\right)$, with $\Delta$ being the sample space, $F$ being $\sigma$-filed, $\left\{F_{t}\right\}_{t \geq 0}$ being the filtration, and $P$ being the probability measure. $h_{i, j}\left(\bar{x}_{i, j}\right), 1 \leq i \leq N$, is an unknown nonlinear smooth function with $h_{i, j}(0)=0$, and $\Phi_{i, j}(\bar{y})$ is a nonlinear uncertainty, which represents the $i^{\text {th }}$ interconnection between the subsystem and other subsystems.

Assumption 1. Since $\Phi_{i, j}(\bar{y})$ and $\psi_{i, j}(\bar{y})$ are smooth functions, unknown smooth functions $\Phi_{i, j, l}\left(y_{l}\right)$ and $\psi_{i, j, l}\left(y_{l}\right)$ satisfy the following inequalities:

$$
\begin{aligned}
\left|\Phi_{i, j}(\bar{y})\right|^{2} & \leq \sum_{l=1}^{N} \Phi_{i, j, l}^{2}\left(y_{l}\right), \\
\left\|\psi_{i, j}(\bar{y})\right\|^{2} & \leq \sum_{l=1}^{N} \psi_{i, j, l}^{2}\left(y_{l}\right),
\end{aligned}
$$

with $\Phi_{i, j, l}(0)=\psi_{i, j, l}(0)=0, l=1,2, \ldots, N$.

Remark 1. Note that $\Phi_{i, j, l}\left(y_{l}\right)$ and $\psi_{i, j, l}\left(y_{l}\right)$ are smooth functions and $\Phi_{i, j, l}(0)=\psi_{i, j, l}(0) ; \quad$ there exist unknown functions $\bar{\Phi}_{i, j, l}\left(y_{l}\right)$ and $\bar{\psi}_{i, j, l}\left(y_{l}\right)$ which can be expressed as 


$$
\begin{aligned}
\left|\Phi_{i, j}(\bar{y})\right|^{2} & \leq \sum_{l=1}^{N} y_{l}^{2} \bar{\Phi}_{i, j, l}^{2}\left(y_{l}\right), \\
\left\|\psi_{i, j}\left(\bar{y}_{i}\right)\right\|^{2} & \leq \sum_{l=1}^{N} y_{l}^{2} \bar{\psi}_{i, j, l}^{2}\left(y_{l}\right) .
\end{aligned}
$$

2.2. Preliminaries' Description. We will present some lemmas, definitions, and basic knowledge in this part; they will be used in the subsequent developments. Consider the stochastic structure

$$
d x(t)=f(x(t)) \mathrm{d} t+g(x(t)) \mathrm{d} \omega,
$$

where $x$ and $\omega$ are the same as defined in (1) and $f(\cdot)$ and $g(\cdot)$ are local Lipschitz functions and satisfy $f(0)=g(0)$.

Lemma 1 (see [35]). For each pair $(x, y) \in R^{2}$, Young's inequality holds

$$
x y \leq \frac{\varepsilon^{a}}{a}|x|^{a}+\frac{1}{b \varepsilon^{b}}|y|^{b},
$$

where $\varepsilon>0, a>1, b>1$, and $(a-1)(b-1)=1$.

Definition 1. (see [36]). For any given $V(x) \in C^{2}$, which is associated with (4), the infinitesimal generator $\mathscr{L}$ is defined as

$$
\mathscr{L} V(x)=\frac{\partial V}{\partial x} f(x)+\frac{1}{2} \operatorname{Tr}\left\{g(x)^{T} \frac{\partial^{2} V}{\partial x^{2}} g(x)\right\} .
$$

$\operatorname{Tr}(A)$ is the trace of a matrix.

Lemma 2. Consider system (4); if there exists a Lyapunov function $V(x) \in C^{2}, \bar{\mu}_{1}$ and $\bar{\mu}_{2}$ are class kappa functions, and two constants $p_{0}>0$ and $q_{0}>0$ such that

$$
\begin{gathered}
\bar{\mu}_{1}(x) \leq V(x) \leq \bar{\mu}_{2}(x), \\
\mathscr{L} V(x) \leq-p_{0} V(x)+q_{0} .
\end{gathered}
$$

Therefore, one conclusion that can be drawn is for (4) and each $x_{0} \in R^{n}$, the solution satisfies

$$
E[V(x)] \leq V\left(x_{0}\right) e^{-p_{0} t}+\frac{p_{0}}{q_{0}}, \quad \forall t>t_{0} .
$$

Definition 2. For any continuous unknown smooth nonlinear function $h_{i, j}(Z)$ over a compact set $\Delta_{Z} \subset R^{q}$, there exist neural networks $W_{i, j}^{* T} \phi(Z)$ such that, for a desired level of accuracy $\varepsilon_{i, j}$,

$$
\begin{gathered}
h_{i, j}(Z)=W_{i, j}^{* T} \phi(Z)+\delta_{i, j}(Z), \quad\left|\delta_{i, j}(Z)\right| \leq \varepsilon, \\
\psi_{i, j}\left(Z_{i, j}\right)=W_{i, j}^{T} \phi_{i, j}\left(Z_{i, j}\right)+\delta_{i, j}\left(Z_{i, j}\right)\left|\delta_{i, j}\left(Z_{i, j}\right)\right|<\varepsilon_{i, j},
\end{gathered}
$$

where $W_{i, j}^{*}$ is the ideal constant weight vector and is defined by

$$
W_{i, j}^{*}=\arg \min _{W \in R^{N}}\left\{\sup _{Z \in \Delta_{Z}}\left|h_{i, j}(Z)-W^{T} \phi_{i, j}(Z)\right|\right\} .
$$

$\delta_{i, j}(Z)$ is the approximation error, $W_{i, j}=\left[w_{1}, \ldots, w_{N}\right]^{T}$ is the weight vector, and $\phi_{i, j}(Z)=\left[\phi_{1}(Z), \ldots, \phi_{N}(Z)\right]^{T}$ is the basis function vector with $N$ being the number of the networks nodes and $N>1$. Radial basis function $\phi_{i}(Z)=\exp \left[-\left(\left(Z-\varsigma_{i}\right)^{T}\left(Z-\varsigma_{i}\right) / \eta_{i}^{2}\right)\right], i=1,2, \ldots, N$, where $\varsigma_{i}=\left[\varsigma_{i 1}, \varsigma_{i 2}, \ldots, \varsigma_{i n}\right]^{T}$, is the center of the receptive field, and $\eta_{i}$ is the width of the Gaussian function. For the $i^{\text {th }}$ subsystem, $W_{i, j}^{T} \phi(Z)$ will be used to construct unknown function $\bar{h}_{i, j}\left(Z_{i, j}\right)$ at step $j$. At last, we design achievable virtual control signals and adaptive laws in the following form:

$$
\begin{aligned}
\alpha_{i, j}\left(Z_{i, j}\right) & =-k_{i, j} z_{i, j}-\frac{1}{2 p_{i, j}^{2}} z_{i, j}^{3} \widehat{\theta}_{i} \phi_{i, j}^{T}\left(Z_{i, j}\right) \phi_{i, j}\left(Z_{i, j}\right), \\
\dot{\hat{\theta}}_{i} & =\sum_{j=1}^{n_{i}} \frac{\omega_{i}}{2 p_{i, j}^{2}} z_{i, j}^{6} \phi_{i, j}^{T}\left(Z_{i, j}\right) \phi_{i, j}\left(Z_{i, j}\right)-\gamma_{i} \widehat{\theta}_{i},
\end{aligned}
$$

where $i=1,2, \ldots, N, j=1,2, \ldots, n_{i}, k_{i, j}, p_{i, j}, \omega_{i}$, and $\gamma_{i}$ are positive design parameters, and $Z_{i, 1}=x_{i, 1}, Z_{i, j}=$ $\left[\bar{x}_{i, j}^{T}, \widehat{\theta}_{i}\right]^{T}\left(j=2, \ldots, n_{i}\right) \quad$ with $\quad \bar{x}_{i, j}=\left[x_{i, 1}, x_{i, 2}, \ldots, x_{i, j}\right]^{T}$. Now, we introduce a change of coordinates as

$$
z_{i, j}=x_{i, j}-\alpha_{i, j-1} \text {, }
$$

with $\alpha_{i, 0}=0$. To begin with the backstepping design procedure, let us define constant $\theta_{i}$ which will be written as

$$
\theta_{i}=\max \left\{\left\|W_{i, j}\right\|^{2}, \quad j=1,2, \ldots, n_{i}\right\}
$$

where $\widehat{\theta}_{i}$ is the estimate of $\theta_{i}$.

\section{Adaptive Neural Control Design}

A neural controller will be constructed for interconnected system (1). At the same time, the adaptive laws will be given in this section.

Step 1. It follows from $z_{i, 1}=x_{i, 1}, z_{i, 2}=x_{i, 2}-\alpha_{i, 1}$ that

$$
d z_{i, 1}=\left(z_{i, 2}+\alpha_{i, 1}+h_{i, 1}+\Phi_{i, 1}(\bar{y})\right) \mathrm{d} t+\psi_{i, 1}^{T}\left(y_{i}\right) \mathrm{d} \omega_{i} .
$$

Establish a Lyapunov candidate $V_{i, 1}$ as

$$
V_{i, 1}=\frac{1}{4} z_{i, 1}^{4}+\frac{1}{2 \varpi_{i}} \widetilde{\theta}_{i}^{2},
$$

where $\omega_{i}>0$ are design parameters.

By taking (6) and (16) into account, we have

$$
\begin{aligned}
\mathscr{L} V_{i, 1}= & z_{i, 1}^{3}\left(z_{i, 2}+\alpha_{i, 1}+h_{i, 1}+\Phi_{i, 1}(\bar{y})\right) \\
& +\frac{3}{2} z_{i, 1}^{2} \psi_{i, 1}^{T}\left(y_{i}\right) \psi_{i, 1}\left(y_{i}\right)-\frac{1}{\varpi_{i}} \widetilde{\theta}_{i} \dot{\theta}_{i} .
\end{aligned}
$$




$$
\begin{aligned}
z_{i, 1}^{3} z_{i, 2} & \leq \frac{3}{4} z_{i, 1}^{4}+\frac{1}{4} z_{i, 2}^{4}, \\
z_{i, 1}^{3} \Phi_{i, 1}(\bar{y}) & \leq \frac{3}{4} z_{i, 1}^{4}+\frac{1}{4} \Phi_{i, 1}^{4}(\bar{y}), \\
& \leq \frac{3}{4} z_{i, 1}^{4}+\frac{1}{4} N\left(\sum_{l=1}^{N} y_{l}^{4} \bar{\Phi}_{i, j, l}^{4}\left(y_{l}\right)\right), \\
\frac{3}{2} z_{i, 1}^{2} \psi_{i, 1}^{T}\left(y_{i}\right) \psi_{i, 1}\left(y_{i}\right) & \leq \frac{3}{4} z_{i, 1}^{4}+\frac{3}{4} N \sum_{l=1}^{N} y_{l}^{4} \bar{\psi}_{i, 1, l}^{4}\left(y_{l}\right) .
\end{aligned}
$$

Substituting (19)-(21) into (18), it follows that

$$
\begin{aligned}
\mathscr{L} V_{i, 1} \leq & \frac{3}{4} z_{i, 1}^{4}+\frac{1}{4} z_{i, 2}^{4}+\frac{3}{4} z_{i, 1}^{4}+z_{i, 1}^{3}\left(\alpha_{i, 1}+h_{i, 1}\right) \\
& +\frac{1}{4} N \sum_{l=1}^{N} y_{l}^{4} \bar{\Phi}_{i, j, l}^{4}\left(y_{l}\right)+\frac{3}{4} z_{i, 1}^{4} \\
& +\frac{3}{4} N \sum_{l=1}^{N} y_{l}^{4} \bar{P}_{i, 1, l}^{4}\left(y_{l}\right)-\frac{1}{\varpi_{i}} \widetilde{\theta}_{i} \dot{\theta}_{i} .
\end{aligned}
$$

Step $m\left(2 \leq m \leq n_{i}\right)$. According to the coordinate transformation, one has

$$
\begin{aligned}
d z_{i, m}= & \left(z_{i, m+1}+\alpha_{i, m}+h_{i, m}+\Phi_{i, m}(\bar{y})-\mathscr{L} \alpha_{i, m-1}\right) d t \\
& +\left(\psi_{i, m}\left(y_{i}\right)-\sum_{k=1}^{m-1} \frac{\partial \alpha_{i, m-1}}{\partial x_{i, k}} \psi_{i, m-1}^{T}\left(y_{i}\right)\right) d \omega_{i},
\end{aligned}
$$

where

$$
\begin{aligned}
\mathscr{L} \alpha_{i, m-1}= & \sum_{k=1}^{m-1} \frac{\partial \alpha_{i, m-1}}{\partial x_{i, k}}\left(x_{i, k+1}+h_{i, k}+\Phi_{i, k}(\bar{y})\right) \\
& +\frac{1}{2} \sum_{p, q=1}^{m-1} \frac{\partial^{2} \alpha_{i, m-1}}{\partial x_{i, p} \partial x_{i, q}} \psi_{i, p}^{T}\left(y_{i}\right) \psi_{i, q}\left(y_{i}\right)+\frac{\partial \alpha_{i, m-1} \hat{\widehat{\theta}}_{i}}{\partial \widehat{\theta}_{i}}
\end{aligned}
$$

Construct a stochastic Lyapunov candidate:

$$
\begin{aligned}
V_{m}= & \frac{1}{4} z_{i, m}^{4}+\frac{1}{2 \varrho_{i}} \widetilde{\theta}_{i}^{2}, \\
\mathscr{L} V_{i, m}= & z_{i, m}^{3}\left(z_{i, m+1}-\sum_{k=1}^{m-1} \frac{\partial \alpha_{i, m-1}}{\partial x_{i, k}}\left(x_{i, k+1}+h_{i, k}\right)\right. \\
& \left.-\frac{\partial \alpha_{i, m-1} \dot{\widehat{\theta}}_{i}-\sum_{k=1}^{m-1} \frac{\partial \alpha_{i, m-1}}{\partial x_{i, k}} \Phi_{i, k}(\bar{y})+\alpha_{i, m}(\bar{y})}{}+h_{i, m}+\Phi_{i, m}-\frac{1}{2} \sum_{p, q=1}^{m-1} \frac{\partial^{2} \alpha_{i, m-1}}{\partial x_{i, p} \partial x_{i, q}} \psi_{i, p}^{T}\left(y_{i}\right) \psi_{i, q}\left(y_{i}\right)\right) \\
& +\frac{3}{2} z_{i, m}^{2}\left(\psi_{i, m}\left(y_{i}\right)-\sum_{k=1}^{m-1} \frac{\partial \alpha_{i, m-1}}{\partial x_{i, k}} \psi_{i, k}\left(y_{i}\right)\right)^{T} \\
& \cdot\left(\psi_{i, m}\left(y_{i}\right)-\sum_{k=1}^{m-1} \frac{\partial \alpha_{i, m-1}}{\partial x_{i, k}} \psi_{i, k}\left(y_{i}\right)\right)-\frac{1}{\mathfrak{\omega}_{i}} \widetilde{\theta}_{i} \dot{\theta}_{i} .
\end{aligned}
$$

Furthermore, similar to the derivations from (19) to (21), the following inequalities can be verified easily:

$$
\begin{aligned}
z_{i, m}^{3} z_{i, m+1} & \leq \frac{3}{4} z_{i, m}^{4}+\frac{1}{4} z_{i, m+1}^{4}, \\
-z_{i, m}^{3} \sum_{k=1}^{m-1} \frac{\partial \alpha_{i, m-1}}{\partial x_{i, k}} \Phi_{i, k}(\bar{y}) & \leq \frac{3}{4} z_{i, m}^{4} \sum_{k=1}^{m-1}\left(\frac{\partial \alpha_{i, m-1}}{\partial x_{i, k}}\right)^{(3 / 4)}+\frac{1}{4} N \sum_{k=1}^{m-1} \sum_{l=1}^{N} y_{l}^{4} \bar{\Phi}_{i, j, l}^{4}\left(y_{l}\right), \\
z_{i, m}^{3} \Phi_{i, k}(\bar{y}) & \leq \frac{3}{4} z_{i, m}^{4}+\frac{1}{4} N \sum_{l=1}^{N} y_{l}^{4} \bar{\Phi}_{i, m, l}^{4}\left(y_{l}\right), \\
-\frac{1}{2} z_{i, m}^{3} \sum_{p, q=1}^{m-1} \frac{\partial^{2} \alpha_{i, m-1}}{\partial x_{i, p} \partial x_{i, q}} \psi_{i, p}^{T}\left(y_{i}\right) \psi_{i, q}\left(y_{i}\right) & \leq \frac{1}{4} z_{i, m}^{6} \sum_{p=1}^{m-1} \sum_{q=1}^{m-1}\left(\frac{\partial^{2} \alpha_{i, m-1}}{\partial x_{i, p} \partial x_{i, q}}\right)^{2}+\frac{1}{4}(m-1) N \sum_{p=1}^{m-1} \sum_{l=1}^{N} y_{l}^{4} \bar{\psi}_{i, p, l}^{4}\left(y_{l}\right), \\
\frac{3}{2} z_{i, m}^{2}\left\|\psi_{i, m}\left(y_{i}\right)-\sum_{k=1}^{m-1} \frac{\partial \alpha_{i, m-1}}{\partial x_{i, k}} \psi_{i, k}\left(y_{i}\right)\right\|^{2} & \leq \frac{3}{4} m z_{i, m}^{4}\left(1+\sum_{k=1}^{m-1}\left(\frac{\partial \alpha_{i, m-1}}{\partial x_{i, k}}\right)^{4}\right)+\frac{3}{4} m N \sum_{k=1}^{m} \sum_{k=1}^{N} y_{l}^{4} \bar{\psi}_{i, p, l}^{4}\left(y_{i}\right) .
\end{aligned}
$$


Substituting (26)-(30) into (25), it shows the result

$$
\begin{aligned}
\mathscr{L} V_{i, m} \leq & z_{i, m}^{3}\left(\alpha_{i, m}+h_{i, m}-\sum_{k=1}^{m-1} \frac{\partial \alpha_{i, m-1}}{\partial x_{i, k}}\left(x_{i, k+1}+h_{i, k}\right)+\frac{3}{2} z_{i, m}+\frac{3}{4} m z_{i, m}+\frac{3}{4} z_{i, m} \sum_{k=1}^{m-1}\left(\frac{\partial \alpha_{i, m-1}}{\partial x_{i, k}}\right)^{\frac{3}{4}} \frac{1}{4} z_{i, m}^{3} \sum_{p=1}^{m-1} \sum_{q=1}^{m-1}\left(\frac{\partial^{2} \alpha_{i, m-1}}{\partial x_{i, p}}\right)^{2} x_{i, q}\right. \\
& -\frac{\left.\partial \alpha_{i, m-1} \dot{\hat{\theta}}_{i}++\frac{3}{4} m z_{i, m} \sum_{k=1}^{m-1}\left(\frac{\partial \alpha_{i, m-1}}{\partial x_{i, k}}\right)^{4}\right)}{} \\
& +\frac{1}{4} m z_{i, m+1}^{4}+\frac{1}{4}(m-1) N \sum_{p=1}^{m-1} \sum_{l=1}^{N} y_{l}^{4} \bar{\Phi}_{i, j, l}^{4}\left(y_{l}\right)+\frac{1}{4} N \sum_{k=1}^{m} \sum_{k=1}^{N} y_{l}^{4} \bar{\Phi}_{i, k, l}^{4}\left(y_{l}\right)-\frac{1}{\omega_{i}} \tilde{\theta}_{i} \dot{\theta}_{i}+\frac{3}{4} m N \sum_{k=1}^{m} \sum_{l=1}^{N} y_{l}^{4} \bar{\Phi}_{i, k, l}^{4}\left(y_{l}\right) .
\end{aligned}
$$

Step $n_{i}$. By using (5) and the Itô formula, we have

$$
\begin{aligned}
d z_{i, n_{i}}= & \left(u_{i}+h_{i, n_{i}}+\Phi_{i, n_{i}}(\bar{y})-\mathscr{L} \alpha_{i, n_{i}-1}\right) d t \\
& +\left(\psi_{i, n_{i}}\left(y_{i}\right)-\sum_{k=1}^{n_{i}-1} \frac{\partial \alpha_{i, n_{i}-1}}{\partial x_{i, k}} \psi_{i, k}^{T}\left(y_{i}\right)\right) d \omega_{i},
\end{aligned}
$$

where $\mathscr{L} \alpha_{i, n_{i}-1}$ is given in (18). Define a stochastic Lyapunov function $V_{i, n_{i}}$ as

$$
V_{i, n_{i}}=\frac{1}{4} z_{i, n_{i}}^{4}+\frac{1}{2 \varpi_{i}} \widetilde{\theta}_{i}^{2}
$$

Then, by means of (6), we can derive

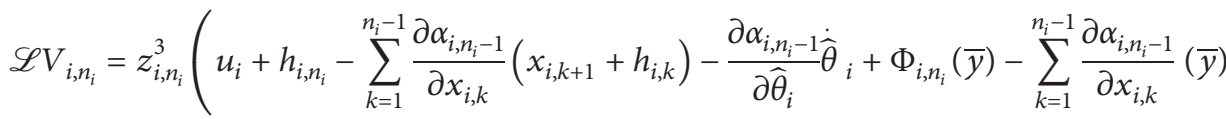

$$
\begin{aligned}
& \left.-\frac{1}{2} \sum_{p, q=1}^{n_{i}-1} \frac{\partial^{2} \alpha_{i, n_{i}-1}}{\partial x_{i, p} \partial x_{i, q}} \psi_{i}^{T}\left(y_{i, p}\right) \psi_{i}\left(y_{i, p}\right)\right)-\frac{1}{\varpi_{i}} \widetilde{\theta}_{i} \dot{\theta}_{i} \\
& +\frac{3}{2} z_{i, n_{i}}^{2}\left\|\psi_{i, n_{i}}\left(y_{i}\right)-\sum_{k=1}^{n_{i}-1} \frac{\partial \alpha_{i, n_{i}-1}}{\partial x_{i, k}} \psi_{i, k}\left(y_{i}\right)\right\|^{2}
\end{aligned}
$$

Repeating similar methods used in the above procedure, we have

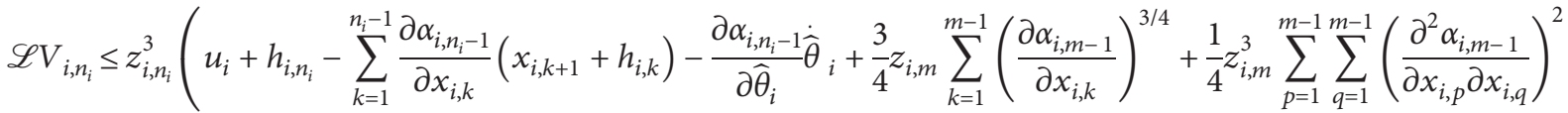

$$
\begin{aligned}
& \left.+\frac{3}{4} z_{i, n_{i}}+\frac{3}{4} n_{i} z_{i, n_{i}} \sum_{k=1}^{n_{i}-1} \frac{\partial \alpha_{i, n_{i}-1}}{\partial x_{i, k}}\left(x_{i, k+1}+h_{i, k}\right)^{4}\right) \\
& +\frac{1}{4} N \sum_{k=1}^{n_{i}} \sum_{l=1}^{N} y_{l}^{4} \bar{\Phi}_{i, j, l}^{4}\left(y_{l}\right)+\frac{3}{4} n_{i} z_{i, n_{i}}-\frac{1}{\omega_{i}} \widetilde{\theta}_{i} \dot{\theta}_{i}+\frac{1}{4}\left(n_{i}-1\right) N \sum_{p=1}^{n_{i}-1} \sum_{l=1}^{N} y_{l}^{4} \bar{\psi}_{i, p, l}^{4}\left(y_{l}\right)+\frac{3}{4} n_{i} N \sum_{k=1}^{n_{i}} \sum_{l=1}^{N} y_{l}^{4} \bar{\psi}_{i, p, l}^{4}\left(y_{l}\right) .
\end{aligned}
$$


Choose $V$ as a Lyapunov function for the whole system:

$$
V=\sum_{i=1}^{N} \sum_{j=1}^{n_{i}} V_{i, j}=\sum_{i=1}^{N}\left(\frac{1}{4} \sum_{j=1}^{n_{i}} z_{i, j}^{4}+\frac{1}{2 \omega_{i}} \tilde{\theta}_{i}^{2}\right) \text {. }
$$
hat

Combining inequalities (22) and (31) with (32), it follows

$$
\begin{aligned}
\mathscr{L} V \leq & \sum_{i=1}^{N} z_{i, 1}^{3}\left(\alpha_{i, 1}+h_{i, 1}+\frac{1}{4} N z_{i, 1} \sum_{l=1}^{N} \sum_{s=1}^{n_{l}} \sum_{k=1}^{s} \bar{\Phi}_{l, k, i}^{4}(\bar{y})+\frac{9}{4} z_{i, 1}+\frac{1}{4} N z_{i, 1} \sum_{l=1}^{N} \sum_{s=1}^{n_{i}-1} s \sum_{p=1}^{s} \bar{\psi}_{l, p, i}^{4}(\bar{y})\right. \\
& \left.+\frac{3}{4} N z_{i, 1} \sum_{l=1}^{N} \sum_{s=1}^{n_{i}-1} s \sum_{p=1}^{s} \bar{\psi}_{l, k, i}^{4}(\bar{y})\right)+\frac{3}{4}\left(j+\frac{7}{3}\right) z_{i, j} \\
& +\sum_{i=1}^{N} \sum_{j=2}^{n_{i}-1} z_{i, j}^{3}\left(\alpha_{i, j}+\frac{1}{4} z_{i, j}^{3} \sum_{p=1}^{j-1} \sum_{q=1}^{j-1}\left(\frac{\partial^{2} \alpha_{i, j-1}}{\partial x_{i, p} \partial x_{i, q}}\right)^{2}\right)+h_{i, 1}+\frac{3}{4} z_{i, j}+\frac{3}{4} z_{i, j} \sum_{k=1}^{j-1}\left(\frac{\partial \alpha_{i, j-1}}{\partial x_{i, k}}\right)^{3 / 4} \\
& -\sum_{i=1}^{N} \frac{1}{\Theta_{i}} \tilde{\theta}_{i} \dot{\hat{\theta}}_{i}-\sum_{k=1}^{j-1} \frac{\partial \alpha_{i, j-1}}{\alpha x_{i, k}}\left(x_{i, k+1}+h_{i, k}\right)+\frac{3}{4} j z_{i, j} \sum_{k=1}^{j-1}\left(\frac{\partial \alpha_{i, j-1}}{\partial x_{i, k}}\right)^{4},
\end{aligned}
$$

$\frac{1}{4} N \sum_{i=1}^{N} \sum_{s=1}^{n_{i}} s \sum_{k=1}^{s} \sum_{l=1}^{N} y_{l}^{4} \bar{\Phi}_{i . k . l}^{4}\left(y_{l}\right)=\frac{1}{4} N \sum_{i=1}^{N} \sum_{l=1}^{N} \sum_{s=1}^{n_{i}} s \sum_{k=1}^{s} y_{i}^{4} \bar{\Phi}_{l, k, i}^{4}\left(y_{i}\right)$,

$\frac{3}{4} N \sum_{i=1}^{N} \sum_{s=1}^{n_{i}} s \sum_{k=1}^{s} \sum_{l=1}^{N} y_{l}^{4} \bar{\psi}_{i . k . l}^{4}\left(y_{l}\right)=\frac{3}{4} N \sum_{i=1}^{N} \sum_{l=1}^{N} \sum_{s=1}^{n_{i}} s \sum_{k=1}^{s} y_{i}^{4} \bar{\psi}_{l, k, i}^{4}\left(y_{i}\right)$

$\frac{1}{4} N \sum_{i=1}^{N} \sum_{s=1}^{n_{i}-1} s \sum_{p=1}^{s} \sum_{l=1}^{N} y_{l}^{4} \bar{\psi}_{i, p, l}^{4}\left(y_{l}\right)=\frac{1}{4} N \sum_{i=1}^{N} \sum_{l=1}^{N} \sum_{s=1}^{n_{i}-1} s \sum_{p=1}^{s} y_{i}^{4} \bar{\psi}_{l, p, i}^{4}\left(y_{i}\right)$.

By (12) and rearranging the sequence, it follows that

$$
\begin{aligned}
-\sum_{i=1}^{N} \sum_{j=2}^{n_{i}} z_{i, j}^{3} \frac{\partial \alpha_{i, j-1} \widehat{\theta}_{i}}{\partial \widehat{\theta}_{i}}= & -\sum_{i=1}^{N} \sum_{j=2}^{n_{i}} z_{i, j}^{3} \frac{\partial \alpha_{i, j}-1}{\partial \widehat{\theta}_{i}}\left(\sum_{k=1}^{n_{i}} \frac{\Phi_{i}}{2 p_{i, k}^{2}} z_{i, k}^{6} \phi_{i, k}^{T} \phi_{i, k}-\gamma_{i} \widehat{\theta}_{i}\right) \\
= & \sum_{k=1}^{j-1} \frac{\Phi_{i}}{2 p_{i, k}^{2}} z_{i, k}^{6} \phi_{i, k}^{T} \phi_{i, k}-\sum_{i=1}^{N} \sum_{j=2}^{n_{i}} z_{i, j}^{3} \frac{\partial \alpha_{i, j-1}}{\partial \widehat{\theta}_{i}} \sum_{k=j}^{n_{i}} \frac{\Phi_{i}}{2 p_{i, k}^{2}} z_{i, k}^{6} \phi_{i, k}^{T} \phi_{i, k} \\
\sum_{i=1}^{N} \sum_{j=2}^{n_{i}} z_{i, j}^{3} \frac{\partial \alpha_{i, j-1}}{\partial \widehat{\theta}_{i}} \gamma_{i} \widehat{\theta}_{i}-\sum_{i=1}^{N} \sum_{j=2}^{n_{i}} z_{i, j}^{3} \frac{\partial \alpha_{i, j-1}}{\partial \widehat{\theta}_{i}} \leq & \sum_{i=1}^{N} \sum_{j=2}^{n_{i}} z_{i, j}^{3} \frac{\partial \alpha_{i, j-1}}{\partial \widehat{\theta}_{i}} \gamma_{i} \widehat{\theta}_{i}-\sum_{i=1}^{N} \sum_{j=2}^{n_{i}} z_{i, j}^{3} \frac{\partial \alpha_{i, j-1}}{\partial \widehat{\theta}_{i}} \sum_{k=1}^{j-1} \frac{\Phi_{i}}{2 p_{i, k}^{2}} z_{i, k}^{6} \phi_{i, k}^{T} \phi_{i, k} \\
& +\sum_{i=1}^{N} \sum_{j=2}^{n_{i}} \frac{\omega_{i}}{2 p_{i, j}^{2}} z_{i, j}^{6}\left(\sum_{k=2}^{j}\left|z_{i, k}^{3} \frac{\partial \alpha_{i, k-1}}{\partial \widehat{\theta}_{i}}\right|\right) .
\end{aligned}
$$


Substituting (38) into (36) yields

$$
\mathscr{L} V \leq \sum_{i=1}^{N} z_{i}^{3}\left(\alpha_{i, 1}+\bar{h}_{i, 1}\left(Z_{i, 1}\right)\right)+\sum_{i=1}^{N} \sum_{j=2}^{n_{i}-1} z_{i, j}^{3}\left(\alpha_{i, j}+\bar{h}_{i, j}\left(Z_{i, j}\right)\right)+\sum_{i=1}^{N} z_{i, n_{i}}^{3}\left(u_{i}+\bar{h}_{i, n_{i}}\left(Z_{i, n_{i}}\right)\right)-\frac{3}{4} \sum_{i=1}^{N} \sum_{j=1}^{n_{i}} z_{i, j}^{4}-\sum_{i=1}^{N} \frac{1}{\Phi_{i}} \widetilde{\theta}_{i} \dot{\hat{\theta}}_{i}
$$

The function $\bar{h}_{i, j}\left(Z_{i, j}\right), i=1,2, \ldots, N$, is defined as

$$
\begin{aligned}
& \bar{h}_{i, 1}\left(Z_{i, 1}\right)=h_{i, 1}+3 y_{i}+\frac{1}{4} N y_{i} \sum_{l=1}^{N} \sum_{s=1}^{n_{i}} \sum_{k=1}^{s} \bar{h}_{l, k, i}^{4}\left(y_{i}\right)+\frac{3}{4} N y_{i} \sum_{i=1}^{N} \sum_{s=1}^{n_{i}} s \sum_{k=1}^{s} \bar{\psi}_{l, k, i}^{4}\left(y_{i}\right)+\frac{1}{4} N y_{i} \sum_{l=1}^{N} \sum_{s=1}^{n_{i}} s \sum_{p=1}^{s} \bar{\psi}_{l, p, i}^{4}\left(y_{i}\right), \\
& \bar{h}_{i, j}\left(Z_{i, j}\right)=h_{i, j}-\sum_{k=1}^{j-1} \frac{\partial \alpha_{i, j-1}}{\partial x_{i, k}}\left(x_{i, k+1}+h_{i, k}\right)+\frac{3}{4} z_{i, j}+\frac{3}{4} z_{i, j} \sum_{k=1}^{j-1}\left(\frac{\partial \alpha_{i, j-1}}{\partial x_{i, k}}\right)^{3 / 4}+\frac{3}{4} j z_{i, j} \sum_{k=1}^{j-1}\left(\frac{\partial \alpha_{i, j-1}}{\partial x_{i, k}}\right)^{4} \\
& +\frac{1}{4} z_{i, j}^{3} \sum_{p=1}^{j-1} \sum_{q=1}^{j-1}\left(\frac{\partial^{2} \alpha_{i, j-1}}{\partial x_{i, p} \partial x_{i, q}}\right)^{2}+\frac{3}{4}(j+2) z_{i, j} \\
& +\frac{\partial \alpha_{i, j-1}}{\partial \widehat{\theta}_{i}} \gamma_{i} \widehat{\theta}_{i}-\sum_{k=1}^{j-1} \frac{\lambda_{i}}{2 p_{i, k}^{2}} z_{i, k}^{6} \phi_{i, k}^{T} \phi_{i, k}+\frac{1}{4} z_{i, j}+\frac{\oplus_{i}}{2 p_{i, j}^{2}} z_{i, j}^{3}\left(\sum_{k=2}^{j}\left|z_{i, k}^{3} \frac{\partial \alpha_{i, k-1}}{\partial \widehat{\theta}_{i}}\right|\right), \quad j=2, \ldots, n_{i}-1, \\
& \bar{h}_{i, n_{i}}\left(Z_{i, n_{i}}\right)=h_{i, n_{i}}-\sum_{k=1}^{n_{i}-1} \frac{\partial \alpha_{i, n_{i}-1}}{\partial x_{i, k}}\left(x_{i, k+1}+h_{i, k}\right)+\frac{\Phi_{i}}{2 p_{i, n_{i}}^{2}} z_{i, n_{i}}^{3}\left(\sum_{k=2}^{n_{i}}\left|z_{i, k}^{3} \frac{\partial \alpha_{i, k-1}}{\partial \widehat{\theta}_{i}}\right|\right)+\frac{3}{4} z_{i, n_{i}} \sum_{k=1}^{n_{i}-1}\left(\frac{\partial \alpha_{i, n_{i}-1}}{\partial x_{i, k}}\right)^{3 / 4} \\
& +\frac{1}{4} z_{i, n_{i}}+\frac{1}{4} z_{i, n_{i}}^{3} \sum_{p=1}^{n_{i}-1} \sum_{q=1}^{n_{i}-1}\left(\frac{\partial^{2} \alpha_{i, n_{i}-1}}{\partial x_{i, p} \partial x_{i, q}}\right)^{2} \\
& +\frac{3}{4}\left(n_{i}+2\right) z_{i, n_{i}}+\frac{\partial \alpha_{i, n_{i}-1}}{\partial \widehat{\theta}_{i}} \gamma_{i} \widehat{\theta}_{i}-\frac{\partial \alpha_{i, n_{i}-1}}{\partial \widehat{\theta}_{i}} \sum_{k=1}^{n_{i}-1} \frac{\omega_{i}}{2 p_{i, k}^{2}} z_{i, k}^{6} S_{i, k}^{T} \phi_{i, k}+\frac{3}{4} n_{i} z_{i, n_{i}} \sum_{k=1}^{n_{i}-1}\left(\frac{\partial \alpha_{i, n_{i}-1}}{\partial x_{i, k}}\right)^{4} .
\end{aligned}
$$

Furthermore, by (10) and Young's inequality, we have

$$
\begin{aligned}
z_{i, j}^{3} \bar{h}_{i, j}\left(Z_{i, j}\right)= & z_{i, j}^{3} \frac{W_{i, j}^{T}}{\left\|W_{i, j}\right\|} \phi_{i, j}\left\|W_{i, j}\right\|+z_{i, j}^{3} \delta_{i, j}\left(Z_{i, j}\right) \leq \frac{1}{2 p_{i, j}^{2}} z_{i, j}^{6}\left\|W_{i, j}\right\|^{2} \phi_{i, j}^{T} \phi_{i, j}+\frac{1}{2} p_{i, j}^{2}+\frac{3}{4} z_{i, j}^{4}+\frac{1}{4} \varepsilon_{i, j}^{4} \leq \frac{1}{2 p_{i, j}^{2}} z_{i, j}^{6} \theta_{i} \phi_{i, j}^{T} \phi_{i, j} \\
& +\frac{1}{2} p_{i, j}^{2}+\frac{3}{4} z_{i, j}^{4}+\frac{1}{4} \varepsilon_{i, j}^{4}, \\
\mathscr{L} V \leq & \sum_{i=1}^{N} z_{i, 1}^{3}\left(\alpha_{i, 1}+\frac{1}{2 p_{i, 1}^{2}} z_{i, 1}^{3} \theta_{i} \phi_{i, 1}^{T} \phi_{i, 1}\right)+\sum_{i=1}^{N} \sum_{j=2}^{n_{i}-1} z_{i, j}^{3}\left(\alpha_{i, j}+\frac{1}{2 p_{i, j}^{2}} z_{i, j}^{3} \theta_{i} \phi_{i, j}^{T} \phi_{i, j}\right)+\sum_{i=1}^{N} z_{i, n_{i}}^{3}\left(u_{i}+\frac{1}{2 p_{i, n_{i}}^{2}} z_{i, n_{i}}^{3} \theta_{i} \phi_{i, n_{i}}^{T} \phi_{i, n_{i}}\right) \\
& +\sum_{i=1}^{N} \sum_{j=1}^{n_{i}}\left(\frac{1}{2} p_{i, j}^{2}+\frac{1}{4} \varepsilon_{i, j}^{4}\right)-\sum_{i=1}^{N} \frac{1}{\varliminf_{i}} \tilde{\theta}_{i} \dot{\hat{\theta}}_{i} .
\end{aligned}
$$


Furthermore, by taking (8) and (9) into account and using the following inequality,

$$
\begin{array}{r}
z_{i, j}^{3} \alpha_{i, j} \leq-a_{i, j} z_{i, j}^{4}-\frac{1}{2 p_{i, j}^{2}} z_{i, j}^{6} \widehat{\theta}_{i} \phi_{i, j}^{T} \phi_{i, j}, \quad i=1,2, \ldots, N, \\
j=1,2, \ldots, n_{i},
\end{array}
$$

(41) can be rewritten as

$$
\begin{aligned}
\mathscr{L} V \leq & -\sum_{i=1}^{N}\left(\sum_{j=1}^{n_{i}} a_{i, j} z_{i, j}^{4}+\frac{\gamma_{i}}{2 \varpi_{i}} \widetilde{\theta}_{i}^{2}\right) \\
& +\sum_{i=1}^{N} \sum_{j=1}^{n_{i}}\left(\frac{1}{2} p_{i, j}^{2}+\frac{1}{4} \varepsilon_{i, j}^{4}+\frac{\gamma_{i}}{2 \varpi_{i}} \theta_{i}^{2}\right) .
\end{aligned}
$$

The inequalities $z_{i, 1}^{3} \alpha_{i, 1} \leq-k_{i, j} z_{i, j}^{4}-\left(\begin{array}{ll}1 / 2 & 2 p_{i, j}^{2}\end{array}\right) z_{i, n_{i}}^{3} \widehat{\theta}_{i} \phi_{i, j}^{T}$ $\phi_{i, j}, \quad i=1,2, \ldots, N, j=1,2, \ldots, n_{i}$, and $\tilde{\theta}_{i} \widehat{\theta}_{i} \leq-(1 / 2) \tilde{\theta}_{i}^{2}+$ $(1 / 2) \theta_{i}^{2}$ have been used in (43).

According to the above design stage, we introduce our main result in the following theorem.

Theorem 1. Under Assumption 1, the closed-loop structure consists of controller (11) and the adaptive law (12), which are designed from system (1). Suppose that, for $1 \leq i \leq N$ and $1 \leq j \leq n_{i}$, all the unknown nonlinear functions can be estimated by the neural network structure with the bounded approximation errors $\varepsilon_{i, j}\left(Z_{i, j}\right)$ in probability. Then, all signals are bounded with the suitable parameter. The error signals $z_{i, j}$ and $\widetilde{\theta}_{i}$ eventually converge to the compact set $\Delta_{s}$ defined by

$$
\Delta_{s}=\left\{z_{i, j}, \widetilde{\theta}_{i}\left|\sum_{i=1}^{N} \sum_{j=1}^{n_{i}} E\left[\left|z_{i, j}\right|^{4}\right] \leq \frac{4 p_{0}}{q_{0}}, \quad\right| \widetilde{\theta}_{i} \mid \leq \sqrt{\frac{2 \omega_{i} p_{0}}{q_{0}}}, 1 \leq i \leq N\right\} .
$$

Proof. Let $p_{0}=\min \left\{4 k_{i, j}, \gamma_{i}, i=1,2, \ldots, N, j=1,2, \ldots, n_{i}\right\}$ and $q_{0}=\sum_{i=1}^{N} \sum_{j=1}^{n_{i}}\left((1 / 2) p_{i, j}^{2}+(1 / 4) \varepsilon_{i, j}^{4}+\left(\gamma_{i} / 2 \Theta_{i}\right) \theta_{i}^{2}\right) ;$ then, (43) can be rewritten as

$$
\mathscr{L} V \leq-p_{0} V+q_{0}, \quad t \geq 0
$$

According to Lemma 1 , it is easy to obtain that $z_{i, j}$ and $\tilde{\theta}$ are bounded. Moreover, in terms of $\theta_{i}$ is a constant, thus $\widehat{\theta}_{i}$ is bounded. It can be derived that $z_{i, j}$ and $\widehat{\theta}_{i}$ are bounded variables. So, $\alpha_{i, j}$ is also bounded in probability. As a result, all signals $x_{i, j}=z_{i, j}+\alpha_{i, j-1}$ are also bounded. Furthermore, from (45), the following inequality holds:

$$
\frac{\mathrm{d} E[V(t)]}{\mathrm{d} t} \leq-p_{0} E[V(t)]+q_{0}
$$

which implies that

$$
E[V(t)] \leq e^{-p_{0} t}[V(0)]+\frac{q_{0}}{p_{0}}, \quad \forall t>0 .
$$

Then, it is easily to obtain that

$$
E[V(t)] \leq \frac{q_{0}}{p_{0}}, \quad t \longrightarrow+\infty .
$$

Therefore, based on the definition of $V$ in (36), the signals $z_{i, j}$ and $\widetilde{\theta}_{i}$ eventually converge to the compact set $\Delta_{s}$ specified in (44). Now, it can be shown that all the signals are semiglobally, uniformly, and ultimately bounded, which are the desired results, and it completes the proof.

\section{Simulation Example}

We will construct a numerical simulation example to verify the effectiveness of the proposed controllers. The stochastic largescale system with nonlinear uncertainty is defined as follows:

$$
\begin{aligned}
d x_{1,1} & =\left(x_{1,2}+h_{1,1}+\Phi_{1,1}\right) \mathrm{d} t+\psi_{1,1}^{T} d w_{i}, \\
d x_{1,2} & =\left(u_{1}+h_{1,2}+\Phi_{1,2}\right) \mathrm{d} t+\psi_{1,2}^{T} d w_{i}, \\
y_{1} & =x_{1,1}, \\
d x_{2,1} & =\left(x_{2,2}+h_{2,1}+\Phi_{2,1}\right) \mathrm{d} t+\psi_{2,1}^{T} d w_{i}, \\
d x_{2,2} & =\left(u_{2}+h_{2,2}+\Phi_{2,2}\right) \mathrm{d} t+\psi_{2,2}^{T} d w_{i}, \\
y_{2} & =x_{2,1},
\end{aligned}
$$

where the nonlinear functions are $h_{1,1}=(3 / 5) x_{1,1}$, $h_{1,2}=3 x_{1,2}^{2}, h_{2,1}=x_{2,1}$, and $h_{2,2}=(2 / 3) x_{2,2}^{2}$, the interconnection functions are $\Phi_{1,1}=(1 / 10)_{1}^{3} \sin \left(x_{1} x_{2}\right), \quad \Phi_{1,2}=$ $2 \sin \left(x_{1} x_{2}\right), \quad \Phi_{2,1}=15 \sin \left(x_{1} x_{2}\right), \quad$ and $\quad \Phi_{2,2}=(1 / 100) x_{1}^{3}$ $\sin \left(x_{2}^{2}\right)$, the stochastic disturbance functions are $\psi_{1,1}=$ $(1 / 15) x_{1}, \psi_{1,2}=(1 / 8) x_{1}, \psi_{2,1}=(1 / 12) x_{1}$, and $\psi_{2,2}=(1 / 8)$ $x_{1}$, and the initial states are chosen as $x_{1,1}(0)=0.2$, $x_{1,2}(0)=0.3, x_{2,1}(0)=0.5, x_{2,2}(0)=0.4$.

According to Theorem 1, the virtual controls $\alpha_{1,1}, \alpha_{2,1}$ and the true control laws $u_{1}, u_{2}$ are chosen, respectively, as

$$
\begin{aligned}
& \alpha_{1,1}=-k_{1,1} z_{1,1}-\frac{1}{2 p_{1,1}^{2}} z_{1,1}^{3} \widehat{\theta}_{1} \phi_{1,1}^{T} \phi_{1,1}, \\
& u_{1}=-k_{1,2} z_{1,2}-\frac{1}{2 p_{1,2}^{2}} z_{1,2}^{3} \widehat{\theta}_{1} \phi_{1,2}^{T} \phi_{1,2}, \\
& \alpha_{2,1}=-k_{2,1} z_{2,1}-\frac{1}{2 p_{2,1}^{2}} z_{2,1}^{3} \widehat{\theta}_{2} \phi_{2,1}^{T} \phi_{2,1}, \\
& u_{2}=-k_{2,2} z_{2,2}-\frac{1}{2 p_{2,2}^{2}} z_{2,2}^{3} \widehat{\theta}_{2} \phi_{2,2}^{T} \phi_{2,2},
\end{aligned}
$$




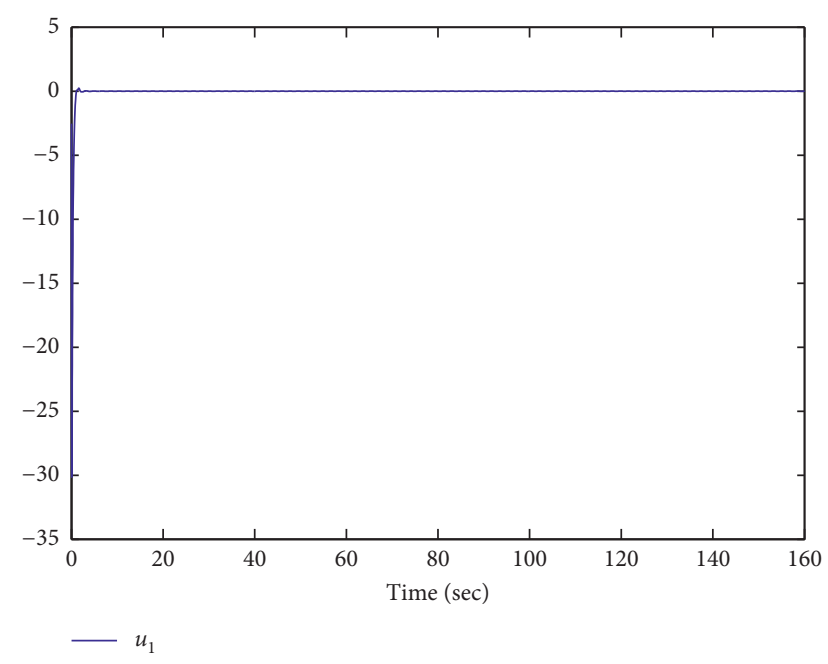

Figure 1: The control input $u_{1}$.

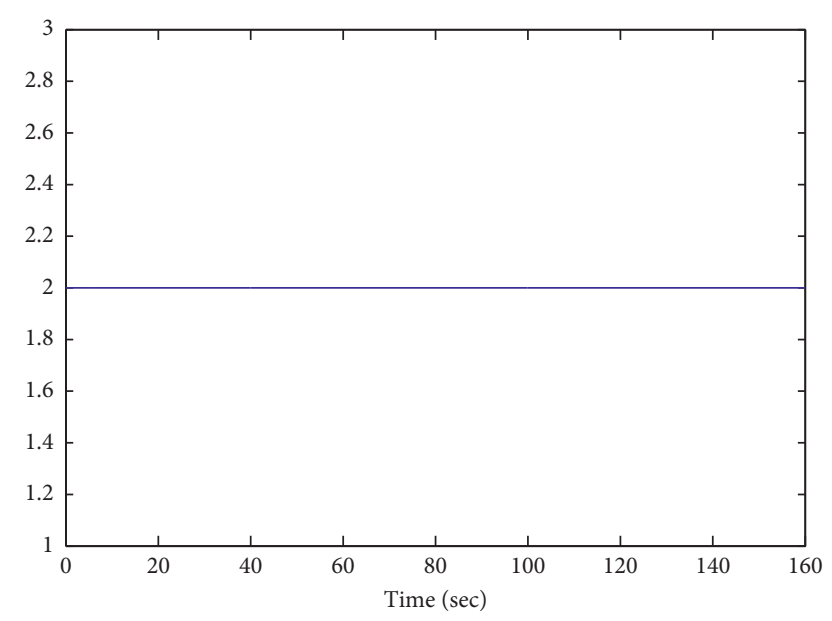

Figure 2: The control input $u_{2}$.

where $\quad z_{1,1}=x_{1,1}, \quad z_{1,2}=x_{1,2}-\alpha_{1,1}, \quad z_{2,1}=x_{2,1}, \quad$ and $z_{2,2}=x_{2,2}-\alpha_{2,1}$. The adaptive laws are given as

$$
\begin{aligned}
& \dot{\hat{\theta}}_{1}=\sum_{j=1}^{2} \frac{\varpi_{1}}{2 k_{1, j}^{2}} z_{1, j}^{6} \phi_{1, j}^{T} \phi_{1, j}-\gamma_{1} \widehat{\theta}_{1}, \\
& \dot{\hat{\theta}}_{2}=\sum_{j=1}^{2} \frac{\Phi_{2}}{2 k_{2, j}^{2}} z_{2, j}^{6} \phi_{2, j}^{T} \phi_{2, j}-\gamma_{2} \widehat{\theta}_{2} .
\end{aligned}
$$

In the simulation, the design parameters are chosen as $k_{1,1}=k_{1,2}=k_{2,1}=k_{2,2}=1.5, \varpi_{1}=\varpi_{2}=1$, and $\gamma_{1}=\gamma_{2}=2$.

The simulation results are illustrated in Figures 1-5, respectively. Figures 1 and 2 demonstrate the control inputs. Figures 3 and 4 illustrate the system states. Figure 5 gives the trajectories of adaptive parameters $\theta_{1}$ and $\theta_{2}$. From Figures $1-5$, it can be seen that all signals in the closed-loop structure are bounded, and the states can track the given

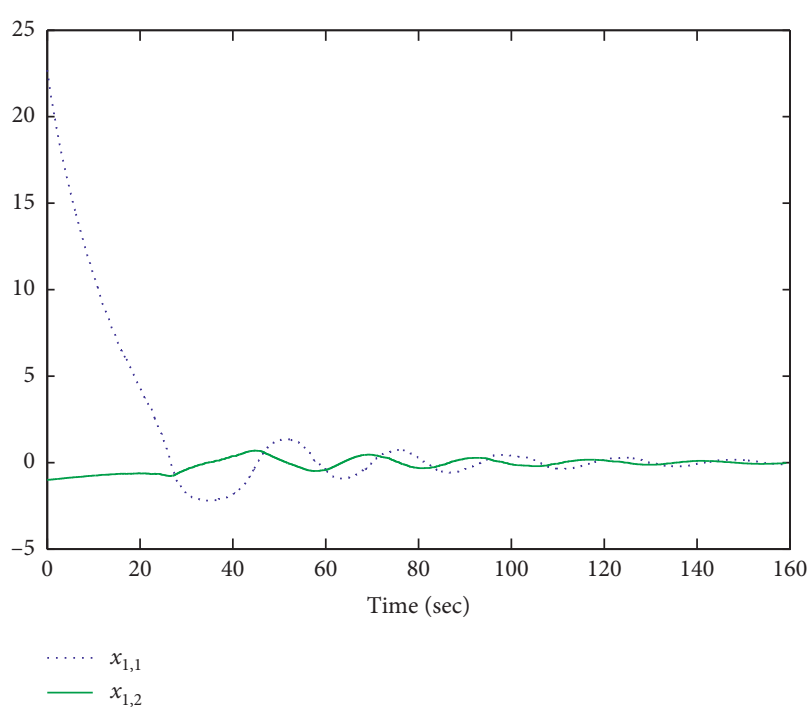

FIGURE 3: The state profile of $x_{1,1}$ and $x_{1,2}$.

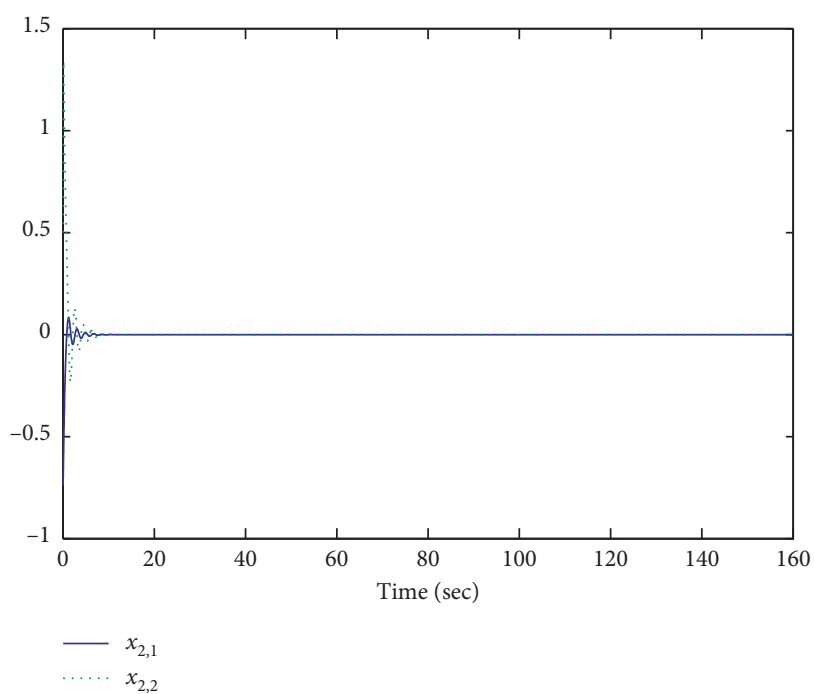

FIgURE 4: The state profile of $x_{2,1}$ and $x_{2,2}$.

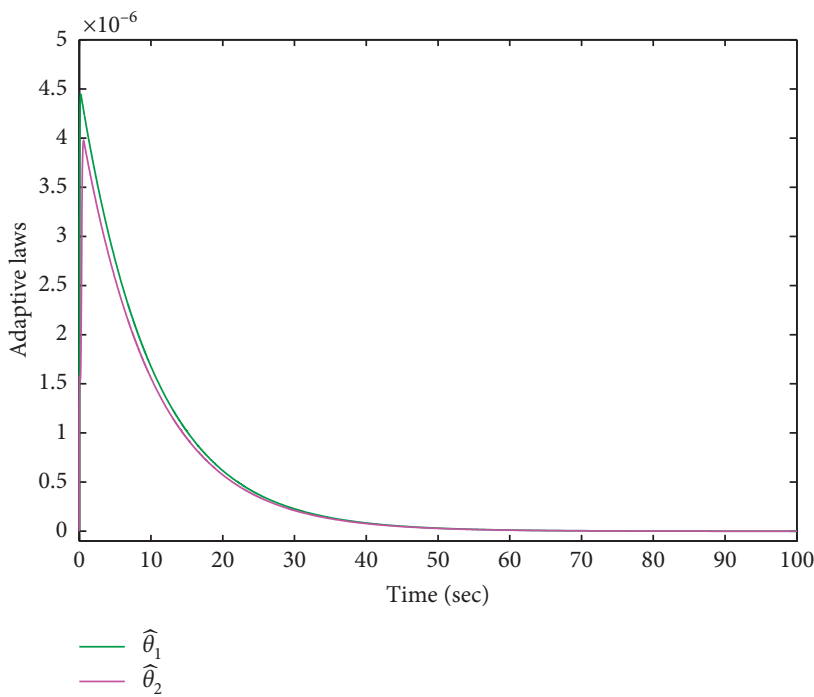

Figure 5: The time profile of $\widehat{\theta}_{1}$ and $\widehat{\theta}_{2}$. 
reference signals. Based on the simulation results, we can conclude that the proposed decentralized control scheme is effective for the large-scale stochastic system.

\section{Conclusions}

Different from the related literature studies, we investigated the control problem for an uncertain interconnected system and designed a state feedback decentralized scheme. There are two difficulties in this paper: one is that the associated terms are completely unknown, and a neural network system is an effective function approximator at present. Therefore, we chose it to estimate these functions. The other is that how to design a decentralized controller to resist the impact among interconnected systems; to deal with this problem, we introduced a neural controller into the control scheme to track the variation of the error. The controller is constructed based on the improved backstepping technique. It is proved that all the signals of the whole closed-loop system are semiglobally, uniformly, and ultimately bounded. The numerical simulations showed that the controller can effectively and steadily drive the original signal. The main novelty is that this work gives a universal formula for establishing a neural state feedback scheme with only one adaptive parameter. Of course, there are still many questions to be further studied. For example, when the solvability of individual subsystems is not assumed, how to find a sufficient condition to make systems stable is an open problem. For another example, we ignored the external disturbance in the system, which is difficult to avoid in the actual control. Therefore, our future work will be devoted to investigating the control method with perturbation terms. Also, we will focus on the control for interconnected systems with output constraints based on the results of this paper.

\section{Data Availability}

The data used to support the findings of this study are included within the article.

\section{Conflicts of Interest}

The authors declare that they have no conflicts of interest.

\section{Acknowledgments}

This work was supported in part by the National Natural Science Foundation of China (Grant nos. 11871117 and 61976027) and the Natural Science Foundation of Liaoning Province of China (Grant nos. 20180551262 and LQ2019008).

\section{References}

[1] C. Wen, "Decentralized adaptive regulation," IEEE Transactions on Automatic Control, vol. 39, no. 10, pp. 2163-2166, 1994.

[2] S. Jain and F. Khorrami, "Decentralized adaptive control of a class of large-scale interconnected nonlinear systems," IEEE Transactions on Automatic Control, vol. 42, no. 2, pp. 136-154, 1997.
[3] S. Jain and F. Khorrami, "Decentralized adaptive output feedback design for large-scale nonlinear systems," IEEE Transactions on Automatic Control, vol. 42, no. 5, pp. 729735, May 1997.

[4] X. Ye and J. Huang, "Decentralized adaptive output regulation for a class of large-scale nonlinear systems," IEEE Transactions on Automatic Control, vol. 48, no. 2, pp. 276-281, 2003.

[5] Z. P. Jiang, "Decentralized and adaptive nonlinear tracking of large-scale systems via output feedback," IEEE Transactions on Automatic Control, vol. 45, no. 11, pp. 2122-2128, 2000.

[6] Z. P. Jiang, D. W. Repperger, and D. J. Hill, "Decentralized nonlinear output-feedback stabilization with disturbance attenuation," IEEE Transactions on Automatic Control, vol. 46, no. 10, pp. 1623-1629, 2001.

[7] Z.-P. Jiang, "Decentralized disturbance attenuating outputfeedback trackers for large-scale nonlinear systems," Automatica, vol. 38, no. 8, pp. 1407-1415, 2002.

[8] S. Tong, C. Liu, Y. Li, and H. Zhang, "Adaptive fuzzy decentralized control for large-scale nonlinear systems with time-varying delays and unknown high-frequency gain sign," IEEE Transactions on Systems, Man, and Cybernetics. Part B (Cybernetics), vol. 41, no. 2, pp. 474-485, 2011.

[9] D. Siljak, "Decentralized control and computations: status and prospects," Annual Review in Automatic Programming, vol. 20, pp. 131-141, 1996.

[10] D. D. Siljak and A. I. Zecevic, "Control of large-scale systems: beyond decentralized feed-back," Annual Reviews in Control, vol. 29, no. 2, pp. 169-179, 2005.

[11] L. Bakule, "Decentralized control: an overview," Annual Reviews in Control, vol. 32, no. 1, pp. 87-98, 2008.

[12] M. Krstic, I. Kanellakopoulos, and P. V. Kokotovic, Nonlinear and Adaptive Control Design, Wiley, New York, NY, USA, 1995.

[13] H.-B. Ji and H.-S. Xi, "Adaptive output-feedback tracking of stochastic nonlinear systems," IEEE Transactions on Automatic Control, vol. 51, no. 2, pp. 355-360, 2006.

[14] S.-J. Liu, S. S. Ge, and J.-F. Zhang, “Adaptive output-feedback control for a class of uncertain stochastic non-linear systems with time delays," International Journal of Control, vol. 81, no. 8, pp. 1210-1220, 2008.

[15] Y.-G. Liu and J.-F. Zhang, "Practical output-feedback risksensitive control for stochastic nonlinear systems with stable zero-dynamics," SIAM Journal on Control and Optimization, vol. 45, no. 3, pp. 885-926, 2006.

[16] S.-J. Liu, J.-F. Zhang, and Z.-P. Jiang, "Decentralized adaptive output-feedback stabilization for large-scale stochastic nonlinear systems," Automatica, vol. 43, no. 2, pp. 238-251, 2007.

[17] X. Su, Z. Li, Y. Feng, and L. Wu, "New global exponential stability criteria for interval-delayed neural networks," Proceedings of the Institution of Mechanical Engineers, Part I: Journal of Systems and Control Engineering, vol. 225, no. 1, pp. 125-136, 2011.

[18] X. Su, P. Shi, L. Wu, and Y. Song, "A novel approach to filter design for T-S fuzzy discrete-time systems with time-varying delay," IEEE Transactions on Fuzzy Systems, vol. 20, no. 6, pp. 1114-1129, 2012.

[19] Q. Zhu, "Stabilization of stochastic nonlinear delay systems with exogenous disturbances and the event-triggered feedback control," IEEE Transactions on Automatic Control, vol. 64, no. 9, pp. 3764-3771, 2019.

[20] B. Chen, X. Liu, K. Liu, and C. Lin, "Direct adaptive fuzzy control of nonlinear strict-feedback systems," Automatica, vol. 45, no. 6, pp. 1530-1535, 2009. 
[21] S. Tong and Y. Li, "Observer-based fuzzy adaptive control for strict-feedback nonlinear systems," Fuzzy Sets and Systems, vol. 160, no. 12, pp. 1749-1764, 2009.

[22] S. Tong, X. He, and H. Zhang, "A combined backstepping and small-gain approach to robust adaptive fuzzy output feedback control," IEEE Transactions on Fuzzy Systems, vol. 17, no. 5, pp. 1059-1069, 2009.

[23] B. Chen, X. Liu, K. Liu, and C. Lin, "Fuzzy-approximationbased adaptive control of strict-feedback nonlinear systems with time delays," IEEE Transactions on Fuzzy Systems, vol. 18, no. 5, pp. 883-892, 2010.

[24] Q. Zhou, P. Shi, J. Lu, and S. Xu, "Adaptive output feedback fuzzy tracking control for a class of nonlinear systems," IEEE Transactions on Fuzzy Systems, vol. 19, no. 5, pp. 972-982, 2011.

[25] H. Zhang, Z. Wang, and D. Liu, "Global asymptotic stability of recurrent neural networks with multiple time-varying delays," IEEE Transactions on Neural Networks, vol. 19, no. 5, pp. 855-873, 2008.

[26] H. Zhang and Y. Quan, "Modeling, identification and control of a class of nonlinear system," IEEE Transactions on Fuzzy Systems, vol. 9, no. 2, pp. 349-354, 2001.

[27] W. Chen, L. Jiao, J. Li, and R. Li, “Adaptive nn backstepping output-feedback control for stochastic nonlinear stict-feedback systems with time-varying delays," IEEE Transactions on Systems, Man, and Cybernetics, Part B (Cybernetics), vol. 40, no. 3, pp. 939-950, 2010.

[28] B. Chen and X. Liu, "Fuzzy approximate disturbance decoupling of MIMO nonlinear systems by backstepping and application to chemical processes," IEEE Transactions on Fuzzy Systems, vol. 13, no. 6, pp. 832-847, 2005.

[29] B. Chen, S. Tong, and X. Liu, "Fuzzy approximate disturbance decoupling of MIMO nonlinear systems by backstepping approach," Fuzzy Sets and Systems, vol. 158, no. 10, pp. 1097-1125, 2007.

[30] Y. Li, C. Yang, S. Ge, and T. Lee, “Adaptive output feedback $\mathrm{nn}$ control of a class of discrete-time mimo nonlinear systems with unknown control directions," IEEE Transactions on Systems, Man, and Cybernetics. Part B (Cybernetics), vol. 41, no. 2, pp. 507-517, 2011.

[31] S. Tong, Y. Li, G. Feng, and T. Li, "Observer-based adaptive fuzzy backstepping dynamic surface control for a class of mimo nonlinear systems," IEEE Transactions on Systems, Man, and Cybernetics. Part B (Cybernetics), vol. 41, no. 4, pp. 1124-1135, 2011.

[32] H. Wang and Q. X. Zhu, "Global stabilization of a class of stochastic nonlinear time-delay systems with SISS inverse dynamics," IEEE Transactions on Automatic Control, 2020.

[33] J. Li, W. Chen, J. Li, and Y. Fang, "Adaptive NN outputfeedback stabilization for a class of stochastic nonlinear strictfeedback systems," ISA Transactions, vol. 48, no. 4, pp. 468-475, Oct. 2009.

[34] J. Li, W. S. Chen, and J. M. Li, "Adaptive NN output-feedback decentralized stabilization for a class of large-scale stochastic nonlinear strict-feedback systems," International Journal of Robust and Nonlinear Control, vol. 21, no. 4, pp. 452-472, 2011.

[35] H. Deng and M. Krstić, "Stochastic nonlinear stabilization-I: a backstepping design," Systems \& Control Letters, vol. 32, no. 3 , pp. 143-150, 1997.

[36] Y. Li, Z. Ma, and S. Tong, "Adaptive fuzzy output-constrained fault-tolerant control of nonlinear stochastic large-scale systems with actuator faults," IEEE Transactions on Cybernetics, vol. 47, no. 9, pp. 2362-2376, 2017. 